\title{
Facial reactions to happy and angry facial expressions: Evidence for right hemisphere dominance
}

\author{
ULF DIMBERG AND MARIA PETTERSON
}

Department of Psychology, Uppsala University, Sweden

\begin{abstract}
Previous research on asymmetric effects of emotional expression and brain-hemispheric asymmetry has supported opposing theories of hemispheric dominance in the control of emotional reactions. In the present study, 32 subjects were exposed to pictures of happy and angry facial stimuli while facial electromyographic (EMG) activity from the zygomatic major and the corrugator supercilii muscle regions was detected from the left and right sides of the face. The subjects reacted spontaneously and rapidly with larger zygomatic EMG activity to happy facial stimuli and larger corrugator EMG activity to angry stimuli. These distinct reactions were significantly larger on the left side of the face. It is concluded that the present results support the hypothesis that the right brain hemisphere is predominantly involved in the control of spontaneously evoked emotional reactions.
\end{abstract}

Descriptors: Facial expression, Emotional reactions, EMG, Hemispheric asymmetry, Asymmetric expression, Positive affect, Negative affect

A great deal of interest has been focused on different aspects of functional asymmetries in the human brain (for reviews see, e.g., Davidson \& Hugdahl, 1995; Hellige, 1993). It has been proposed that emotional processes are controlled asymmetrically by the left and right brain hemispheres.

Research focused on the neurological basis of emotion has also used facial expressions of emotion as another window to the understanding of asymmetrical dominance in emotional processes (e.g., Borod \& Koff, 1990; for a review see, e.g., Camras, Holland, \& Patterson, 1993). The interest in facial expressions is partly due to the fact that the left and right facial muscles are innervated differently by the two hemispheres. More specifically, we know that control of the lower parts of the face is predominantly contralateral, whereas the upper facial muscles are controlled more bilaterally (e.g., Borod \& Koff, 1990; Fridlund, 1994). Thus, particularly for the lower parts of the face, greater left facial muscle activity can be taken as evidence for greater involvement of the right hemisphere and greater right facial muscle activity indicates more left hemisphere dominance.

Results from research in which expression asymmetry and/or hemispheric activation were investigated have not been consistent. In some studies, it had been found that the left side of the face tends to show more extreme expression than the right, indicating right brain dominance (e.g., Borod \& Koff, 1990; Ekman, Hager, \& Friesen, 1981; Sackheim, Gur, \& Saucy, 1978). Other results have shown the opposite, that is, a more intense expression on the

This work was supported by the Swedish Council for Research in the Humanities and Social Sciences.

Address reprint requests to: Ulf Dimberg, Department of Psychology, Uppsala University, Box 1225, S-751 42 Uppsala, Sweden. E-mail: Ulf. Dimberg@psyk.uu.se. right side of the face (e.g., Sirota \& Schwartz, 1982). There are even findings indicating a differentiation between positive and negative emotions (e.g., Davidson, 1993; Schwartz, Ahern, \& Brown, 1979). At least two different hypotheses have been proposed (for a review, see, e.g., Camras et al., 1993; Fridlund, 1988). First, according to the right hemisphere hypothesis, the right hemisphere dominates the mediation of emotion and, therefore, facial expressions should be more intense on the left side of the face. Second, according to the valence hypothesis, the left hemisphere is the seat of positive emotions and the right hemisphere the seat of negative emotions. Consequently, positive emotional expressions should be more intensely expressed on the right side of the face and negative expressions should be more intense on the left side of the face.

It is not clear why the results from the different studies have been inconsistent. One explanation could be that widely different emotional stimuli, such as cognitive manipulations (e.g., Schwartz et al., 1979) and visual stimulation (e.g., Jäncke, 1994), have been used. It is therefore not self-evident that the different types of emotional elicitors are comparable and the inconsistency in findings might be that the subjects, at least in some studies, have not been exposed to an appropriate emotion elicitor (Davidson, 1993).

One type of positive and negative stimuli that has been demonstrated to consistently evoke particular positive and negative emotional response patterns is facial expression stimuli. In a number of studies, subjects were exposed to pictures of different facial expressions of emotion while their own facial electromyographic (EMG) activity from emotion-relevant facial muscles was measured (e.g., Dimberg, 1982; for reviews see Dimberg, 1990, 1997a). It has been found consistently that happy facial stimuli spontaneously evoke increased activity in the zygomatic major muscle (the muscle used when smiling; Hjortsjö, 1970). Angry faces, on the 
other hand, elicit increased activity in the corrugator supercilii muscle region (muscles used to approximate the eyebrows when frowning). These reactions can also be very rapidly evoked. Distinct facial reactions were detected after only $500 \mathrm{~ms}$ of exposure (Dimberg, 1997b; Dimberg \& Thunberg, 1998) and similar rapid reactions can be automatically evoked without the involvement of conscious cognitive processes (Dimberg, Thunberg, \& Elmehed, 2000). In these studies, however, facial EMG was only measured from one side of the face, that is, the left side, providing no information on possible hemispheric dominance.

Consequently, the aim of the present study was to compare left and right hemifacial EMG reactions in subjects exposed to positive and negative facial expression stimuli. More precisely, the subjects were exposed to happy and angry facial stimuli while EMG activity was measured from the left and right zygomatic major muscle and corrugator supercilii muscle regions. According to the right hemisphere hypothesis, the left hemifacial reactions should be larger as compared with the right hemifacial reactions for both happy and angry facial stimuli. According to the valence hypothesis, happy stimuli should evoke larger zygomatic EMG responses on the right side of the face, whereas angry stimuli should evoke larger corrugator EMG responses on the left side of the face. Because the lower facial muscles are innervated contralaterally and the upper face is controlled bilaterally (e.g., Fridlund, 1988), it was expected that the asymmetric effects would be more clear cut for the zygomatic EMG response to happy stimuli.

Furthermore, because earlier studies (e.g., Dimberg, 1997b; Dimberg \& Thunberg, 1998) consistently have demonstrated that distinct facial reactions to facial stimuli are evident already during the first second of exposure, and particularly so during the period $500-1,000 \mathrm{~ms}$ after stimulus onset, the critical effects in the present study were expected to be obtained during this period.

\section{Method}

\section{Subjects}

The subjects were 32 right-handed students attending courses at Uppsala University. There were 16 men and 16 women (mean age $=24$ years). The subjects were paid about US $\$ 10.00$. Handedness was determined by simply asking the subjects if they were right or left handed.

\section{Apparatus and Data Scoring}

Subjects were tested individually. They were seated in a chair in a soundproof experimental chamber with a screen $1.6 \mathrm{~m}$ in front of them onto which slides of facial expressions were projected with a picture size of $25 \times 35 \mathrm{~cm}$. Because it has been found that facial EMG reactions can be influenced by an orienting response when different stimuli are presented intermixed (Dimberg, 1996), one happy and one angry face was presented nonmixed in blocks of six trials. Thus, similar to the design in previous studies which have been successful to detect distinct facial EMG reactions to facial stimuli (e.g., Dimberg, 1982, 1990, 1997b; Dimberg \& Thunberg, 1998), each subject was exposed repeatedly in blocks of six trials to one happy and one angry face. The slides were eight angry and eight happy faces selected from Ekman and Friesen (1976). Consequently, the slides were distributed among the 32 subjects so that only 4 subjects were exposed to identical happy and angry stimuli. The order of presentation of blocks of happy and angry stimuli was counterbalanced so that half of the group saw an angry face first and the other half saw a happy face first. The duration of exposure was $8 \mathrm{~s}$. The intertrial intervals varied between 25 and $35 \mathrm{~s}($ mean $=$ $30 \mathrm{~s})$.

Facial muscle activity was measured using Beckman miniature $\mathrm{Ag} / \mathrm{AgCl}$ electrodes, filled with Beckman electrode paste. The electrodes were attached on both the left and right sides of the face, over the zygomatic major and the corrugator supercilii muscle regions (Fridlund \& Cacioppo, 1986). The skin was cleaned with alcohol and rubbed with electrode paste. This procedure reduced the electrode site impedance to less than $10 \mathrm{k} \Omega$. The raw EMG activity was measured with Coulbourn Hi Gain Amplifiers bandpass filtered from 10 to $1000 \mathrm{~Hz}$. The raw EMG was integrated by Coulbourn Contour Following Integrators with a time constant set at $20 \mathrm{~ms}$. The integrated signals were digitized using a 12 bit A/D converter and were stored on a PC with a sampling frequency of $100 \mathrm{~Hz}$. The data were expressed in microvolts.

Phasic facial EMG responses were scored and averaged over intervals of $100 \mathrm{~ms}$ during the first second of exposure. The responses were expressed as change in activity from the prestimulus level, which was defined as the mean activity during the last second before stimulus onset.

\section{Procedure}

So that subjects would be blind to the purpose of the study, they were told that their facial sweat gland activity would be measured. After the facial electrodes were attached, the subjects were instructed to sit in a relaxed fashion and watch the pictures. After the experiment and an interview in which they were asked if they realized that facial muscle activity had been measured, the subjects were informed about the true purpose of the study. None of the subjects reported having deduced what had actually been measured.

\section{Design and Statistical Analysis}

The basic design was a three factorial with emotion (angry face vs. happy face), side of the face (left vs. right), and trials (six) as repeated-measure factors (e.g., Kirk, 1968). Data were analyzed with analyses of variance (ANOVA). Because the most clear-cut effects were expected to be obtained during the period 5001,000 ms after stimulus onset (e.g., Dimberg, 1997b), the first second after onset was divided in two periods (0-500 and 500$1,000 \mathrm{~ms}$ ) each with five 100 -ms intervals. Thus, period (two) and intervals (five) were added as repeated measures in the ANOVA.

One ANOVA was performed for the left and right zygomatic muscle regions and one for the two corrugator muscle regions. Because repeated-measures $F$ tests are likely to result in positively biased tests, Geisser-Greenhouse conservative $F$ tests were used, reducing the degrees of freedom (e.g., Kirk, 1968). A priori comparisons between means for the left and right side of the face were evaluated by $t$ tests.

\section{Results}

Because there were no significant effects or interaction effects for the Trial factor, this factor will not be discussed. The data for the zygomatic major and the corrugator supercilii muscle regions are given in Figure 1. As can be seen in the upper panel of Figure 1 the overall zygomatic EMG activity increased over periods, $F(1,31)=$ $12.69, p<.01$. Happy faces also evoked overall larger zygomatic EMG activity than did angry stimuli during the 500-1,000-ms period, $t(31)=2.33, p<.02$. However, as can also be seen and as indicated by the Side $\times$ Emotion interaction, $F(1,31)=4.91, p<$ .05 , and the Side $\times$ Emotion $\times$ Period interaction, $F(1,31)=8.45$, 

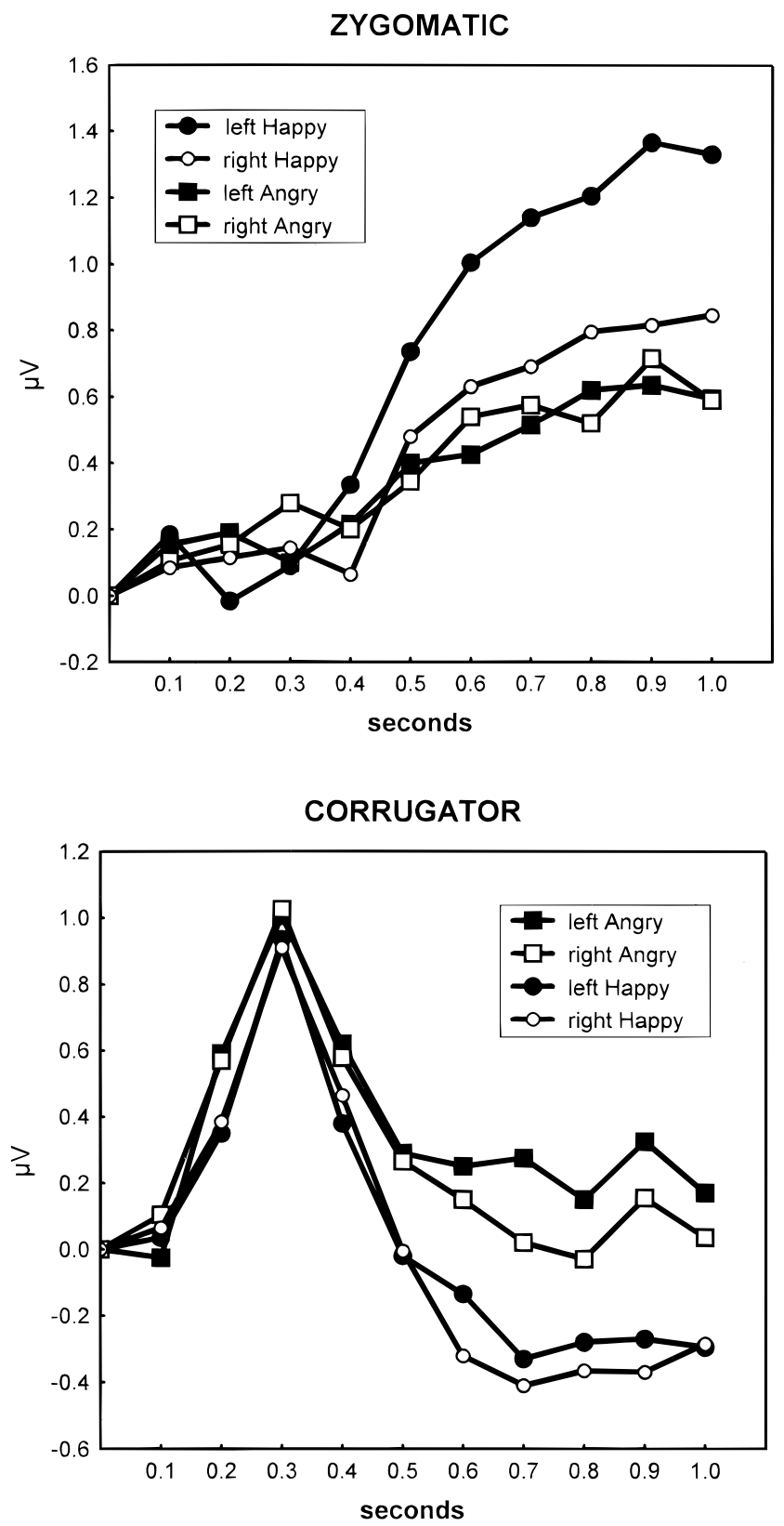

Figure 1. The facial electromyogram (EMG) responses to pictures of happy and angry facial stimuli plotted as a function of $100-\mathrm{ms}$ intervals during the first second after stimulus onset. The upper panel illustrates the data for the left and right zygomatic major muscle regions. The lower panel illustrates the corresponding data for the corrugator supercilii muscle region.

$p<.01$, the zygomatic EMG response was larger to happy faces on the left side as compared with the right side of the face, and this effect was particularly evident during the 500-1,000-ms period, $t(31)=9.48, p<.001$. The corresponding $t$ test for angry stimuli was $t(31)<1$. Finally, it can be noted that even if the difference between happy and angry stimuli was lower on the right than on the left side of the face (see Figure 1), this difference was still significant on the right side of the face, $t(31)=3.44, p<.01$.

The mean response for the corrugator supercilii muscle is given in the lower panel of Figure 1. As can be seen and as indicated by the interval factor $F(4,129)=17.09, p<.001$, and the period factor, $F(1,31)=36.70, p<.001$, the corrugator EMG activity was characterized by an initial distinct increase in response to all stimuli within the first $0-500-\mathrm{ms}$ period. This effect did not differ between the two sides of the face or between the different stimuli. Overall, the corrugator EMG reaction was larger to angry than to happy faces $F(1,31)=7.87, p<.01$. As can be seen in the lower panel of the figure, however, and as indicated by the Period $\times$ Emotion interaction, $F(1,31)=8.13, p<.01$, the larger response to angry faces was particularly evident during the $500-1,000-\mathrm{ms}$ period. Although emotion and side of face did not interact, a planned comparison between means showed that the corrugator EMG response to angry stimuli was larger on the left side than on the right side of the face, $t(31)=2.83, p<.01$, which can also be seen clearly during the 500-1,000-ms period in the lower panel of the Figure 1 . The corresponding $t$ test for happy stimuli was $t(31)=$ $1.48, n s$.

\section{Discussion}

Consistent with earlier studies (e.g., Dimberg, 1982, 1990), subjects spontaneously and rapidly reacted with a larger zygomatic major muscle reaction to happy faces and a larger corrugator supercilii muscle reaction to angry faces, which was most clear cut after $500 \mathrm{~ms}$ of exposure (e.g., Dimberg \& Thunberg, 1998). Most importantly, the present results demonstrated that the facial reactions were larger on the left side of the face. This finding was true for both the zygomatic EMG response to happy faces and for the corrugator EMG response to angry faces. Consequently, the present results favors the right hemisphere hypothesis. That is, this hypothesis predicted larger facial muscle activity on the left side of the face, in particular for positive reactions. The valence hypothesis, however, predicted larger muscle activity on the right side of the face for positive emotions. In this respect, the results were opposite to those predicted by the valence hypotheses.

As in earlier studies in which rapid facial EMG responses have been studied (e.g., Dimberg, 1997b), the initial corrugator supercilii EMG response in the present study was characterized by increased activity during the first period of exposure with a peak after about $300 \mathrm{~ms}$. Interestingly, and also consistent with earlier studies, this early response component was similar to all the different stimuli (e.g., Dimberg, 1997b), indicating that it does not reflect the emotional content of the stimuli but rather a nonspecific effect of visual stimulation. One explanation could be that this response is an effect of movements and orienting activity. It is therefore interesting that this initial response did not differ either as a function of stimuli or as a function of side of face. This fact further underscores that the response reflects a nonspecific effect of visual stimulation without any emotional processing concomitant with differential hemispheric control.

It has been suggested that the left and right side of the face may differ in muscle strength (e.g., Fridlund, 1988). Even if it is not self-evident that this should be the case, it could be useful to include a nonaffective control condition that would assure that the present results do not simply reflect difference in muscle strength. However, one finding in the present study is clearly against this interpretation. That is, as noted above the initial corrugator EMG reaction reflected a nonaffective response and the fact that this response component was similar on both sides of the face indicate that the left and right sides are similar in muscle strength.

Another question is whether the facial reactions are evoked spontaneously or reflect voluntarily controlled actions. A number of factors suggest these responses are spontaneous. First, the sub- 
jects were instructed to sit calm and relaxed and they were not aware that the muscle activity was being measured. Furthermore, the facial reactions were very rapidly evoked and looked similar to the responses detected in earlier studies (e.g., Dimberg \& Thunberg, 1998). In particular, the facial reactions looked almost identical both in shape and rapidity to those obtained when subjects were, by help of the backward masking technique, unconsciously exposed to happy and angry faces (Dimberg et al., 2000).

In the present study, it was predicted that the difference between the left and right facial muscle reactions might be more clear cut for the zygomatic than for the corrugator muscles. To some extent the present data support this prediction. That is, overall, zygomatic EMG responses to happy faces and corrugator EMG responses to angry faces were significantly larger on the left side of the face when evaluated by a priori $t$ tests. On the other hand, the laterality effect for corrugator EMG activity was not strong enough to produce a significant interaction between emotion and side of face.

The present results are not consistent with the results from earlier studies in which electroencephalography (EEG) was measured from the left and right sides of the brain. In those studies (e.g., Davidson, 1993; Davidson et al., 1990), it was found that positive emotion was accompanied by higher activity in the left brain hemisphere, indicating left brain hemisphere dominance for positive emotions. Thus, a straightforward way to further elucidate this question would be to measure both facial EMG and EEG using a paradigm similar to the present one. In that way it would be possible to simultaneously study asymmetric effects for both facial EMG reactions and brain activity.

\section{REFERENCES}

Borod, J. C., \& Koff, E. (1990). Lateralization for facial emotional behavior: A methodological perspective. International Journal of Psychology, 25, 157-177.

Camras, L. A., Holland, E. A., \& Patterson, M. J. (1993). Facial expression. In M. Lewis \& J. M. Haviland (Eds.), Handbook of emotions (pp. 199208). New York: Guilford Press.

Davidson, R. J. (1993). The neuropsychology of emotion and affective style. In M. Lewis \& J. M. Haviland (Eds.), Handbook of emotions (pp. 143-154). New York: Guilford Press.

Davidson, R. J., Ekman, P., Saron, C. D., Senulis, J. A., \& Friesen, W. V. (1990). Approach/withdrawal and cerebral asymmetry: Emotional expression and brain physiology. I. Journal of Personality and Social Psychology, 58, 330-341.

Davidson, R. J., \& Hugdahl, K. (1995). Brain asymmetry. Cambridge, MA: MIT Press.

Dimberg, U. (1982). Facial reactions to facial expressions. Psychophysiology, 19, 643-647.

Dimberg, U. (1990). Facial electromyography and emotional reactions (Award address for distinguished early career contribution to psychophysiology, 1988). Psychophysiology, 27, 481-494.

Dimberg, U. (1996). Facial EMG and the orienting response. Psychophysiology, 33, S34.

Dimberg, U. (1997a). Psychophysiological reactions to facial expressions. In U. Segerstråle \& P. Molnar (Eds.), Nonverbal communication: Where nature meets culture (pp. 47-60). Mahwah, NJ: Erlbaum.

Dimberg, U. (1997b). Facial reactions: Rapidly evoked emotional responses. Journal of Psychophysiology, 11, 115-123.

Dimberg, U., \& Thunberg, M. (1998). Rapid facial reactions to different emotionally relevant stimuli. Scandinavian Journal of Psychology, 39, $39-45$.

Dimberg, U., Thunberg, M., \& Elmehed, K. (2000). Unconscious facial reactions to emotional facial expressions. Psychological Science, 11, 86-89.
Ekman, P., \& Friesen, W. (1976). Pictures of facial affect. Palo Alto, CA: Consulting Psychologists Press.

Ekman, P., Hager, J. C., \& Friesen, W. V. (1981). The symmetry of emotional and deliberate facial actions. Psychophysiology, 18, 101-106.

Fridlund, A. J. (1988). What can asymmetry and laterality in EMG tell us about the face and brain? International Journal of Neuroscience, 39 , $53-69$.

Fridlund, A. J. (1994). Human facial expressions: An evolutionary view. New York: Academic Press.

Fridlund, A. J., \& Cacioppo, J. T. (1986). Guidelines for human electromyographic research. Psychophysiology, 23, 567-589.

Hellige, J. B. (1993). Hemispheric asymmetry: What's right and what's left. Cambridge, MA: Harvard University Press.

Hjortsjö, C. H. (1970). Man's face and mimic language. Malmö, Sweden: Nordens Boktryckeri.

Jäncke, L. (1994). An EMG investigation of the coactivation of facial muscles during the presentation of affect-laden stimuli. Journal of Psychophysiology, 8, 1-10.

Kirk, R. E. (1968). Experimental design: Procedures for the behavioral sciences. Belmont, CA: Wadsworth.

Sackheim, H. A., Gur, R. C., \& Saucy, M. C. (1978). Emotions are expressed more intensely on the left side of the face. Science, 202, 434-435.

Schwartz, G. E., Ahern, G. L., \& Brown, S. L. (1979). Lateralized facial muscle response to positive and negative emotional stimuli. Psychophysiology, 16, 561-571

Sirota, A. D., \& Schwartz, G. E. (1982). Facial muscle patterning and lateralization during elation and depression imagery. Journal of Abnormal Psychology, 91, 25-34.

(Received May 12, 1999; Accepted October 26, 1999) 